\title{
ON THE PROXIMAL RELATION BEING CLOSED
}

\author{
HARVEY B. KEYNES ${ }^{1}$
}

1. Introduction. In this paper, we utilize the algebraic techniques suggested in [2] and [3] to investigate some questions about the proximal relation being closed, where the transformation groups have compact Hausdorff phase spaces. In $\$ 2$, a sufficient condition is given for the property of being closed; when the transformation group is pointwise almost periodic, the condition is equivalent to distal. The problem of whether the proximal relation being closed is preserved by a transformation group homomorphism is considered in $\S 3$. In the pointwise almost periodic case, it is shown that the proximal relation itself is actually preserved, and, a fortiori, the closure is preserved. In the general case, a sufficient condition is given for the closure to be preserved.

Let $(Z, T)$ be a transformation group with a uniform phase space $Z$. If $x, y \in Z$, then $x$ and $y$ are proximal if for every index $\alpha$ of $Z$, there exists $t \in T$ such that $(x t, y t) \in \alpha$. The proximal relation, denoted by $P(Z)$, is the set of all pairs of proximal points. It is known [3] that $P(Z)$ is reflexive symmetric and $T$-invariant but in general is not an equivalence relation or closed. If $z \in Z$, then $z P(Z)$ denotes the set $[w \mid(z, w) \in P(Z)]$. The transformation group $(Z, T)$ is distal if every pair of distinct points is not proximal.

Let $(W, T)$ be another transformation group. A transformation group homomorphism from $(Z, T)$ to $(W, T)$ is a continuous map $f: Z \rightarrow W$ such that $f \pi^{t}=\pi^{t} f$, for all $t \in T$ [3]. If $f$ is onto, we write $f:(Z, T) \underset{\rightarrow}{\rightarrow}(W, T)$. We denote the set of all transformation group homomorphisms from $(Z, T)$ to itself by $\operatorname{End}(Z, T)$.

Let $Z$ now be a compact Hausdorff space. The enveloping semigroup, denoted by $E(Z)$, is defined to be $\left[\pi^{t} \mid t \in T\right]-\subset Z^{Z}$, providing $Z^{Z}$ with its product topology. As a general reference, see [2]. The class of minimal right ideals will be denoted by $\mathfrak{T}_{E(Z)}$, and the set of idempotents in $\operatorname{UT}_{E(Z)}$ will be denoted by $I(Z)$.

Throughout this paper, $(X, T)$ and $(Y, T)$ will denote transformation groups with compact Hausdorff phase spaces $X$ and $Y$. We will utilize right-handed functional notation. As a general reference, see $[4]$.

Received by the editors July 11, 1966.

1 These results form a portion of a doctoral dissertation written at Wesleyan University while the author was a National Science Foundation Cooperative Graduate Fellow. 
The author would like to thank Professors Robert Ellis and Walter Gottschalk for illuminating conversations concerning the contents of this paper.

2. On the proximal relation being closed. The following lemma will be utilized throughout the paper.

(2.1) Lemma. Suppose that there exist $p \in M \in \mathfrak{N}_{E(X)}$ such that $p$ is continuous. Then the following statements are equivalent:

(1) $P(X)$ is an equivalence relation.

(2) $P(X)$ is closed.

Proof. That (2) implies (1) follows from [1, Corollary 1]. We show that (1) implies (2). Since $P(X)$ is an equivalence relation, $\mathfrak{T}_{E(X)}=[M]\left[2\right.$, Theorem 2]. Let $\left(\left(x_{n}, y_{n}\right)\right)$ be a net in $P(X)$ such that $\left(x_{n}, y_{n}\right) \rightarrow(x, y)$. By [2, Remark 6], for all $n$ and for all $q \in M$, $x_{n} q=y_{n} q$. Let $p$ be a continuous element of $M$. Since for all $n, x_{n} p$ $=y_{n} p$ and $\left(x_{n} p, y_{n} p\right) \rightarrow(x p, y p)$, it follows that $x p=y p$. By $[3$, Lemma 4], $(x, y) \in P(X)$. The result follows.

(2.2) Theorem. Suppose that there exist $p \in M \in \mathscr{M}_{E(X)}$ such that $p$ is continuous and for all $N \in \mathfrak{M}_{E(X)}, p N \cap N p \neq \varnothing$. Then $P(X)$ is closed.

Proof. Choose $p \in M \in \mathfrak{M}_{E(X)}$ satisfying the hypotheses. By (2.1) and [2, Theorem 2], we need only show that $\mathfrak{\Re}_{E(X)}=[M]$.

Let $N \in \mathscr{M}_{E(X)}$. Since $p N \in \mathscr{M}_{E(X)}[2$, Remark 3] and $p N \subset M N$ $\subset M$, then $p N=M$. Thus, $N p \cap M \neq \varnothing$. But $N p \subset N M \subset N$. It follows that $M \cap N \neq \varnothing$ and $M=N$. The proof is completed.

Note that we have shown that if $p \in M \in \mathfrak{M}_{E(X)}$ and for all $N \in \mathfrak{M}_{E(X)}, p N \cap N p \neq \varnothing$, then $P(X)$ is an equivalence relation. The converse is clear.

(2.3) Corollary. Suppose that there exists $M \in \mathbb{M}_{E(X)}$ such that $M \cap \operatorname{End}(X, T)$ is nonvacuous. Then $P(X)$ is closed.

Proof. Let $p \in M \cap \operatorname{End}(X, T)$. Since $p$ is continuous and for all $t \in T, p \pi^{t}=\pi^{t} p$, it follows that for all $q \in E(X), p q=q p$. Now use (2.2).

(2.4) Corollary. Suppose that $T$ is abelian, and there exist $p \in M \in \mathfrak{M}_{E(X)}$ such that $p$ is continuous. Then $P(X)$ is closed.

Proof. Since $T$ is abelian, if $p \in E(X)$ and $p$ is continuous, then $p \in \operatorname{End}(X, T)$.

As an example of (2.4), consider the sliding circle. 
Now let $p \in M \in \mathscr{M}_{E(X)}$ such that $p M=M p$. If $I(M)$ denotes the set of idempotents in $M$, it is known that $(M u \mid u \in I(M))$ is a partition of $M$ into subgroups. If $u \in I(M)$ and $p \in M u$, it follows that $M=M u$ and $M$ is a group under composition. If, in addition, for all $N \in \mathfrak{M}_{E(X)}, p N=N p$, then $\mathfrak{T}_{E(X)}=[M]$ and $I(X)$ is a singleton.

When $(X, T)$ is a pointwise almost periodic transformation group, we can modify (2.2) as follows:

(2.5) Theorem. Let $(X, T)$ be a pointwise almost periodic transformation group. Then the following statements are equivalent:

(1) There exist $p \in M \in \mathscr{M}_{E(X)}$ such that $p$ is continuous and for all $N \in \mathfrak{M}_{E(X)}, p N=N p$.

(2) There exist $p \in M \in \mathscr{M}_{E(X)}$ and $N \in \mathscr{T}_{E(X)}$ such that $p N=N p$.

(3) $(X, T)$ is a distal transformation group.

Proof. It is clear that (1) implies (2). We show that (2) implies (3). Let $p \in M \in \mathscr{T}_{E(X)}$ and $N \in \mathscr{T}_{E(X)}$ such that $p N=N p$. Since $p N \subset M E \subset M$ and $N p \subset N$, then $M \cap N \neq \varnothing$ and $M=N$. Thus $p M=M p$. It follows that $M$ is a group and has a unique idempotent.

Let $N \in \mathfrak{T}_{E(X)}$. Since there is a set isomorphism between the idempotents of $M$ and $N$ [2, Proposition 1], $N$ has a unique idempotent.

Let $x \in X$. Then $x P(X)=x I(X)$. Let $N \in \mathscr{N}_{E(X)}$ and let $I(N)$ denote the idempotents in $N$. By [2, Theorem 1], $x \in x I(N)$. Thus,

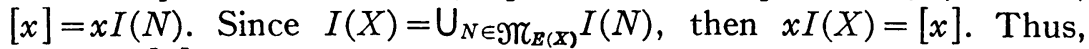
$x P(X)=[x]$, and $(X, T)$ is a distal transformation group.

We show that (3) implies (1). If $(X, T)$ is a distal transformation group, it is known that $\mathfrak{T}_{E(X)}=[E(X)]$. Thus, id $=\pi^{e} \in E(X)$ and id satisfies (1). The proof is completed.

By using the comment in (2.4), we obtain from (2.5):

(2.6) Corollary. Let $(X, T)$ be a pointwise almost periodic transformation group. Let $T$ be abelian. Then the following statements are equivalent:

(1) There exists $p \in M \in \mathfrak{M}_{E(X)}$ such that $p$ is continuous.

(2) $(X, T)$ is a distal transformation group.

3. The preservation of the closure of the proximal relation under a homomorphism. We shall first consider this problem for the pointwise almost periodic case. The following two lemmas are crucial to the proof.

(3.1) Lemma. Let $f:(X, T) \underset{\rightarrow}{\sim}(Y, T)$, and $g:(E(X), T) \underset{\rightarrow}{\rightarrow}(E(Y), T)$ be induced by $f[2$, Lemma $4(1)]$. Then $I(X) g=I(Y)$.

Proof. Since $\mathfrak{T}_{E(X)} g=\mathfrak{T}_{E(Y)}$ and $g$ is a semigroup homomorphism, it is clear that $I(X) g \subset I(Y)$. We show that $I(Y) \subset I(X) g$. Let 
$v \in I(Y)$. It is direct to verify that $v g^{-1}$ is a nonvacuous compact semigroup. Since $\mathfrak{T}_{E(X)} g=\mathfrak{T}_{E(Y)}$, there exists $M \in \mathfrak{T}_{E(X)}$ such that $v \in M g$. Then $v g^{-1} \cap M$ is a nonvacuous compact semigroup. There exists an idempotent $u \in v g^{-1} \cap M[3$, Lemma 3]. Then $u \in I(X)$ and $u g=v$. The result follows.

(3.2) Lemma. Let $A(X)=[x \mid x \in X$ and $T$ is almost periodic at $x]$. Then for all $x \in X, A(X) \cap x P(X)=x I(X)$.

Proof. Let $x \in X$. We show that $A(X) \cap x P(X) \subset x I(X)$. Let $y \in A(X) \cap x P(X)$. Choose $M \in \mathscr{N}_{E(X)}$ such that for all $p \in M, x p=y p$. Let $I(M)$ denote the set of idempotents in $M$. Since $y \in A(X)$, then $y \in y I(M)[2$, Theorem 1]. Let $u \in I(M)$ such that $y=y u$. Then $y=y u=x u \in x I(M) \subset x I(X)$. We show that $x I(X) \subset A(X) \cap x P(X)$. Let $y \in x I(X)$. There exists $u \in I(X)$ such that $y=x u$. Then $y u=x u^{2}=x u=y$. It follows that $y \in x P(X)$ and, by [2, Theorem 1], $y \in A(X)$. The proof is completed.

We now prove the main result of this section.

(3.3) Theorem. Let $f:(X, T) \underset{\rightarrow}{\rightarrow}(Y, T)$. Let $\hat{f}: X \times X \rightarrow Y \times Y$ be defined by $(x, y) \hat{f}=(x f, y f)$. Let $(Y, T)$ be a pointwise almost periodic transformation group. Then $P(X) \hat{f}=P(Y)$.

Proof. It is direct to verify that we need only show that for all $x \in X, x P(X) f=(x f) P(Y)$. Let $x \in X$. By [3, Lemma 2], $x P(X) f$ $\subset(x f) P(Y)$. Let $A(X)$ and $g$ be defined as in (3.2) and (3.1) respectively. By (3.2), $A(X) \cap x P(X)=x I(X)$. Then $x P(X) f \supset x I(X) f$ $=(x f)(I(X) g)=(x f) I(Y)$ by (3.1). Since $(Y, T)$ is a pointwise almost periodic transformation group, $(x f) I(Y)=(x f) P(Y)$. Thus, $x P(X) f$ $\supset(x f) P(Y)$. The proof is completed.

The conclusion of (3.3) is not generally true; see [3, Example 2].

Since $X$ and $Y$ are compact Hausdorff spaces and $\hat{f}$ is continuous, we obtain:

(3.4) Corollary. Let $f:(X, T) \underset{\rightarrow}{\rightarrow}(Y, T)$. Let $(Y, T)$ be a pointwise almost periodic transformation group. Suppose that $P(X)$ is closed. Then $P(Y)$ is closed.

As another application of (3.2) to the proximal relation, we have the following generalization of [1, Theorem 4].

(3.5) Theorem. Let $x \in X$. Let $M \in \mathfrak{M}_{E(X)}$. Let $I(M)$ and $A(X)$ be defined as in (3.2). Then

(1) $A(X) \cap x P(X)=x I(X)$,

(2) For all $y, z \in x I(M),(y, z) \in P(X)$. If $y \in A(X)$ and for all $z \in x I(M),(y, z) \in P(X)$, then $y \in x I(M)$. 
(3) Let $q \in M$ such that for all $x \in X,(x, x q) \in P(X)$. Then $q \in I(M)$.

Proof. (1) is just (3.2). The proof of (2) proceeds as in [1, Theorem 4] using (1), since $y \in A(X)$. To show (3), note that $x q \in A(X)$. By using (2) to show that there exists $v \in I(M)$ such that $x q=x v$, the proof in $[1$, Theorem 4$]$ holds. The result follows.

When $(Y, T)$ is not a pointwise almost periodic transformation group, it is not known whether the conclusion of (3.4) is always valid. We now give a condition which does yield the conclusion without additional hypotheses.

The following lemma is of some independent interest.

(3.6) Lemma. Let $f:(X, T) \underset{\rightarrow}{\rightarrow}(Y, T)$. Let $g:(E(X), T) \underset{\rightarrow}{\rightarrow}(E(Y), T)$ be induced by $f$. Let $p \in E(X)$ such that $p$ is continuous. Then $p g$ is continuous.

Proof. Let $y \in Y$. Let $\mathcal{F}$ be an ultra-filter on $Y$ such that $\mathcal{F} \rightarrow y$. By [3, Lemma 7], it is sufficient to show that $\mathcal{F}(p g) \rightarrow y(p g)$.

Now $F^{-1}$ is a filter on $X$. Let $g$ be an ultra-filter on $X$ such that

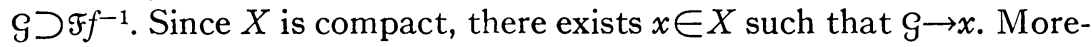
over, $\mathcal{G} f \supset \mathcal{F f}^{-1} f=\mathfrak{F}$, whence $\mathcal{G} f=\mathcal{F}$. Thus, $\mathcal{F}=\mathcal{G} f \rightarrow x f$, and $x f=y$. Since $p$ is continuous, $\mathscr{F}(p g)=\mathcal{G} f(p g)=S p f \rightarrow x p f=(x f) p g=y(p g)$. The result follows.

(3.7) Theorem. Let $f:(X, T) \underset{(}{\longrightarrow}(Y, T)$. Suppose that $P(X)$ is closed. Let $\mathfrak{M}_{E(X)}=[M]$. Suppose further that there exists $p \in M$ such that $p$ is continuous. Then $P(Y)$ is closed.

Proof. Let $g:(E(X), T) \underset{\rightarrow}{\rightarrow}(E(Y), T)$ be induced by $f$. Choose $p \in M$ such that $p$ is continuous. By (3.6), $p g$ is continuous. Moreover, $p g \in M g \in \mathfrak{M}_{E(Y)} g=\mathfrak{M}_{E(X)}$. By [2, Lemma 7$], P(Y)$ is an equivalence relation. It follows from (2.1) that $P(Y)$ is closed. The proof is completed.

\section{REFERENCES}

1. J. Auslander, On the proximal relation in topological dynamics, Proc. Amer. Math. Soc. 11 (1960), 890-895.

2. R. Ellis, $A$ semigroup associated with a transformation group, Trans. Amer. Math. Soc. 94 (1960), 272-281.

3. R. Ellis and W. Gottschalk, Homomorphisms of transformation groups, Trans. Amer. Math. Soc. 94 (1960), 258-271.

4. W. Gottschalk and G. Hedlund, Topological dynamics, Colloq. Publ., Vol. 36, Amer. Math. Soc., Providence, R. I., 1955.

WESLEYAN UNIVERSITY AND

University of California, Santa Barbara 